\title{
Think tanks españoles: análisis longitudinal de su presencia en medios nacionales e internacionales (2004-2018)
}

\author{
Spanish think tanks: longitudinal analysis of their \\ presence in national and international media \\ (2004-2018)
}

\author{
Vanessa Roger-Monzó; Fernando Castelló-Sirvent
}

Cómo citar este artículo:

Roger-Monzó, Vanessa; Castelló-Sirvent, Fernando (2020). “Think tanks españoles: análisis longitudinal de su presencia en medios nacionales e internacionales (2004-2018)". Profesional de la información, v. 29, n. 3, e290331.

https://doi.org/10.3145/epi.2020.may.31

Artículo recibido el 07-06-2019

Aceptación definitiva: 08-01-2020

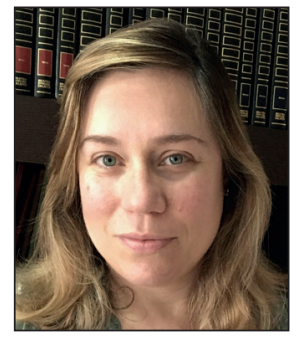

Vanessa Roger-Monzó $\square$ https://orcid.org/0000-0002-7498-0406

ESIC Business \& Marketing School

Avda. Blasco Ibáñez, 55.

46021 Valencia, España

vanessa.roger@esic.edu

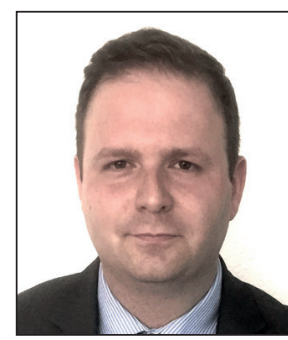

Fernando Castelló-Sirvent https://orcid.org/0000-0002-2088-0039

ESIC Business \& Marketing School

Avda. Blasco Ibáñez, 55.

46021 Valencia, España

fernando.castello@esic.edu

\section{Resumen}

El objetivo de este estudio es explorar la presencia de think tanks españoles en medios de comunicación nacionales e internacionales. Para llevar a cabo la investigación se ha desarrollado un análisis cuantitativo de los contenidos publicados sobre 70 think tanks, durante 15 años (2004-2018). Los contenidos publicados se han obtenido mediante Factiva ${ }^{\circledR}$, una base de datos de Dow Jones \& Company que proporciona acceso a más de 35.000 medios de comunicación de todo el mundo. Los resultados sugieren que la representación mediática de los think tanks aumenta gradualmente a lo largo de los años, con independencia del ciclo económico y de la situación política, llegando a cuadriplicar su presencia en el período analizado. Del mismo modo, se confirma la existencia de un incremento de la hegemonía de unos pocos think tanks en la agrupación de mensajes del debate público y su concentración en torno a unos pocos medios y agencias de medios.

\section{Palabras clave}

Think tanks; Medios de comunicación; Representación mediática; Crisis económica; Políticas públicas; Factiva.

\section{Abstract}

The objective of this study is to explore the presence of Spanish think tanks in national and international media based on a quantitative analysis of content published on 70 think tanks over 15 years (2004-2018). The published content was obtained through $F \mathrm{Fctiva}^{\circledR}$, a database from Dow Jones \& Company, that provides access to more than 35,000 mass media around the world. The results suggest that the representation of think tanks in the media has gradually increased over the years, regardless of the economic cycle or political situation, quadrupling over the analyzed period. Moreover, an increase of the hegemony of a few think tanks in the grouping of messages on the public debate and their concentration in a few media and media agencies are confirmed.

\section{Keywords}

Think tanks; Mass media; Media representation; Economic crisis; Public policy; Factiva. 


\section{Introducción}

El término think tank hace referencia a los actores políticos orientados a producir investigación y análisis para influir y asesorar a la élite política y a la sociedad en general (Misztal, 2012; Stone, 2004; 1996; McGann; Weaver, 2000). Los distintos contextos y la heterogeneidad de estas entidades implican que los términos utilizados para referirse a las mismas sean diversos:

- centros de investigación (Castillo-Esparcia; Guerra-Heredia; Almansa-Martínez, 2017);

- fábricas de ideas (Abelson, 2009);

- coaliciones de defensa (Sherrington, 2000).

La pluralidad existente de think tanks, con grandes diferencias entre ellos, dificulta la existencia de una definición unánime (Abelson, 2012; Medvetz, 2008).

Las áreas de investigación que actualmente abarcan estas entidades incluyen política económica, educativa, energética, de salud, de ciencia, tecnología, social, defensa y seguridad nacional, medioambiente y relaciones internacionales, entre otras (McGann, 2019).

Los think tanks tienen como misión fundamental transformar los debates públicos, dado que se muestran como representantes esenciales en política (McGann, 2007). Lowery y Marchetti (2012) determinan que el interés principal de estas organizaciones radica en la difusión y puesta en marcha de las políticas públicas. Por tanto, su función como actores políticos se apoya en la enunciación y difusión de ideas (Montobbio, 2013). Además, resulta relevante su papel como interlocutores, mediadores o intermediarios de ideas y, en consecuencia, organizadores de debates en la agenda pública (McGann; Viden; Rafferty, 2014; Urrutia, 2013).

\subsection{Definición y evolución de los think tanks}

Su naturaleza heterogénea determina la existencia de diversas definiciones, en función de la perspectiva empleada. La definición más conocida es la de McGann y Weaver (2000), para quienes los think tanks son organizaciones no gubernamentales y sin ánimo de lucro, dedicadas a la investigación, con independencia organizativa respecto del gobierno y de los intereses de la sociedad, como empresas, grupos de interés y partidos políticos. Según James son

"organizaciones independientes dedicadas a la investigación multidisciplinar con la intención de influir en las políticas públicas" (James, 1993, p. 492).

Por su parte, Castillo-Esparcia (2009) los define como entidades que articulan su discurso a través de la investigación y el análisis, empleando la divulgación de sus propuestas como una fuente de acción política, dado que sus estrategias de comunicación directa o indirecta permiten ejercer influencia en la opinión pública. Para Rich (2004) son organizaciones independientes y sin ánimo de lucro que, basadas en la experiencia y las ideas, tratan de influir en el proceso de formulación de políticas. Por su parte, Chuliá establece que los think tanks son

“organizaciones privadas, sin ánimo de lucro, dedicadas a transferir a la sociedad datos y análisis sobre asuntos de interés público, con el objetivo de mejorar las condiciones de información y conocimiento en las que los responsables políticos y los ciudadanos adoptan sus decisiones" (Chuliá, 2018, p. 333).

Sin embargo, estas definiciones no se adecuan al contexto europeo, dado que existen diferentes patrones de interacción entre think tanks y partidos políticos: en países como Alemania, Holanda y España existe una gran vinculación entre think tanks y partidos, predominando la cooperación entre ambos tipos de instituciones. Por el contrario, en Estados Unidos y Canadá, estas entidades presentan un elevado nivel de autonomía respecto de los partidos, lo que los convierte en competidores en el "mercado de ideas" (Baier; Bakvis, 2001).

En este sentido, la definición de Requejo-Coll et al. (2000) se presenta más consistente con la evidencia empírica que presenta el caso español, ya que considera que los think tanks son grupos organizados de forma privada o con el apoyo de la Administración, dedicados a la investigación, aunque no a su implementación, comunicando sus conclusiones a través de artículos, libros, congresos, conferencias o apariciones puntuales en los medios de comunicación, tanto orientadas al público en general como a públicos específicos. Además, destacan que pueden estar afiliados a instituciones académicas, constituidos como fundaciones sin ánimo de lucro, estar promovidos por profesionales, o ser dependientes de partidos políticos o grupo de interés.

Si bien muchos think tanks dependen de alguna institución (Arshed, 2017), de las definiciones anteriores se desprende una visión idealizada como instituciones independientes que brindan información objetiva y neutral para favorecer el debate público (Shaw et al., 2015). No obstante, se constata que la dependencia de estas entidades descansa en tres pilares (Medvetz, 2012):

- el acceso político para impactar en el debate público;

- el acceso a actores económicos para obtener apoyo financiero;

- el acceso a los medios de comunicación para lograr visibilidad en la agenda mediática.

Se incorpora a estos pilares el factor académico como elemento de reputación, puesto que se asienta en el prestigio de la investigación. En la conexión entre investigación y política, y en la capacidad de los think tanks de producir conoci- 
miento, es donde reside el origen de su poder público (Wiarda, 2008). Sin embargo, el compromiso con el rigor académico queda subordinado a los conflictos de intereses políticos y de financiación (Parrilla; Almiron; Xifra, 2016).

La democratización, la internacionalización y la evolución tecnológica son algunas de las principales razones que explican la proliferación de estas entidades en todo el mundo (Ponsa-Herrera, 2014). McGann (2019) señala que han aumentado en número, alcance e impacto de trabajo a nivel mundial y destaca que poseen un gran potencial para sostener gobiernos democráticos.

Por otra parte, la existencia de diversas categorías de think tanks, ha llevado a los expertos a proponer "tradiciones" nacionales o regionales. En esta línea, Stone (2005) describe una tradición anglo-estadounidense en la que son organizaciones independientes que colaboran en el análisis de cuestiones políticas al margen del gobierno y de los partidos políticos. En la tradición asiática, se encuentran habitualmente integrados en las propias corporaciones, son semi-independientes y a menudo poseen una estrecha interacción con el gobierno o con figuras políticas individuales.

Por lo que respecta a España, la primera iniciativa orientada a desarrollar un sistema constante de información para la toma de decisiones públicas tiene su origen en 1877, con la creación del Instituto Geográfico y Estadístico. No obstante, la dictadura franquista supuso un obstáculo para la instauración de think tanks. Durante sus años de vigencia se crearon diversos centros de pensamiento, aunque todos ellos al servicio del régimen. Esta circunstancia ha supuesto que, a diferencia de otros países de su entorno, España tuviera una escasa tradición de estas entidades (Ponsa-Herrera; González-Capitel, 2015).

La transición española favoreció que los partidos políticos se consolidaran como instrumentos esenciales para la participación política. El contexto democrático establecido a partir de la transición dio lugar al sistema preponderante de think tanks en España, caracterizado por la cultura de la subvención, la nula presencia de filantropía privada y la alta dependencia de los partidos políticos. En este sentido,

“la proliferación de fundaciones vinculadas orgánicamente a los partidos políticos -sobre todo a partir de 1994, año de la creación de las subvenciones públicas específicas para las fundaciones de los partidos por parte del Estado- significó la consolidación de estas organizaciones como tipología dominante de think tanks" (Ponsa-Herrera; González-Capitel, 2015, p. 24).

Desde finales del siglo XX, los think tanks en España aumentaron su número debido al incremento de la participación del país en asuntos internacionales (Parrilla; Almiron; Xifra, 2016), consolidándose como nuevos elementos políticos para el asesoramiento, autoridad y firme control en la toma de decisiones políticas (Saura-Casanova, 2015), con una mayor presencia social, política y comunicativa (Castillo-Esparcia; Guerra-Heredia; Almansa-Martínez, 2017). Actualmente, el modelo español se encuentra

"fuertemente polarizado entre las fundaciones de partidos políticos, que obtienen la mayor parte de la financiación pública, y las organizaciones que funcionan a la manera de asociaciones empresariales, sustentándose a partir de las cuotas de las empresas representadas en el Patronato" (Ponsa-Herrera; González-Capitel, 2015, p. 28).

Siguiendo a McGann (2019), España tenía registrados en 2018 un total de 66 think tanks, ocupando la posición 21 en la lista de países con mayor número de estas organizaciones. De ellos, 19 aparecen en el ranking: nueve se sitúan entre los más influyentes de Europa Occidental y diez como más relevantes en las áreas de tecnología, seguridad, defensa y medioambiente, entre otras.

\subsection{Think tanks: comunicación e influencia}

Una de las funciones esenciales de los think tanks es informar a sus públicos, que se concretan, fundamentalmente, en decisores públicos y en medios de comunicación (Xifra, 2005). Estas entidades desarrollan investigaciones y promueven sus hallazgos como hitos dignos de ser ampliamente difundidos en los medios (Posner, 2001). Así, una de sus funciones fundamentales es alcanzar una amplia cobertura e influir en los discursos de los medios, al considerarse como la principal maniobra para impactar en las decisiones políticas (Lalueza; Girona, 2016; Rich; Weaver, 2000) y medir su efectividad (Abelson, 2012).

Tradicionalmente se ha asociado una mayor visibilidad mediática a mayores recursos (Danielian, 1989), aunque este aspecto no contempla que algunos pueden convertirse en una fuente obligatoria al tratar ciertos temas. (Rich; Weaver, 2000). Además, se ha detectado que los periodistas tienden a utilizar constantemente a expertos que aparecen con mayor regularidad en los medios de comunicación, circunstancia que reduce la pluralidad (Graber, 1993).

El estudio de Lalueza y Girona (2016) sobre los think tanks durante la crisis económica señala que estos se decantan por apropiarse de un nicho temático para incrementar su impacto en los medios de comunicación y se convierten en una fuente de referencia en ese tema. En el contexto de la economía española, esta estrategia es la utilizada por la Fundación de Estudios de Economía Aplicada (Fedea) y de la Fundación de Cajas de Ahorros (Funcas). También en este mismo campo, otra de las estrategias orientadas a obtener elevada visibilidad en los medios pasa por proponer un amplio abanico de soluciones que permitan superar las consecuencias de la crisis económica, abarcando distintas áreas, tales como empleo, impuestos o pensiones, entre otras, tal y como sucede con el caso de Círculo de Empresarios. Además, en tanto que los discursos se presentan cohesionados, se produce una mayor difusión por parte de los medios de comunicación, facilitando la creación y el desarrollo de estados de opinión en el debate público (Davis, 2012). 
Por otra parte, la influencia de los think tanks sobre el discurso de los medios de comunicación también está determinada por la percepción que estos tengan de la relación de dependencia entre un think tank y un partido político, tendiendo a perder influencia en medios cuanto mayor sea la dependencia percibida (Lalueza; Girona, 2016).

Si tomamos en consideración la propuesta de Baier y Bakvis (2001) sobre el análisis de las relaciones entre think tanks y partidos, se constata la cercanía del modelo español a la experiencia alemana, puesto que los partidos políticos han conformado los think tanks con los que mantienen una gran dependencia. Este hecho contrasta con el modelo estadounidense o de Canadá, en el que existe una manifiesta separación entre las distintas entidades, y donde los think tanks ejercen principalmente su influencia sobre el público a través de los medios de comunicación. Entre ambos extremos, Inglaterra ocupa un lugar intermedio. No obstante en España también existe un gran número de think tanks financiados por instituciones privadas y empresas.

En lo referente a los ciclos políticos, se distinguen tres etapas (Selee, 2013):

- la primera, cuando se acotan las principales ideas o asuntos a tratar;

- la segunda, cuando se ofrecen alternativas;

- la tercera, cuando se materializa la toma de decisiones.

De este modo, los think tanks deben combinar diferentes estrategias comunicativas con los actores implicados en cada una de las fases con el fin de lograr la mayor influencia posible.

El término think tank otorga a estas organizaciones la legitimidad necesaria para alcanzar la representación mediática (Denham, 2005) que, a su vez, les brinda credibilidad para desempeñar acciones que se materialicen en políticas públicas (McDonald, 2014). No obstante, los resultados de diversos estudios destacan que son muy pocos los que tienen presencia destacada en la agenda mediática, puesto que en la mayoría de los casos suele prevalecer el contacto directo con actores políticos relevantes (Lalueza; Girona, 2016; Ponsa-Herrera, 2014; Barberà; Arregui, 2011). En ciertas situaciones como una crisis económica, la necesidad de implementar políticas de forma urgente supone que esas instituciones recurran a medidas con resultados más inmediatos como, por ejemplo, estrategias de lobby político (Leeson; Ryan; Williamson, 2012). A pesar de ello, se constata la importancia de los medios de comunicación para difundir sus premisas y conocimientos (Abelson, 2012; Rich; Weaver, 2000) o para lograr una imagen de prestigio entre políticos y líderes de opinión (Urrutia, 2013).

Las investigaciones empíricas que analizan la influencia real de estas entidades en el discurso mediático son escasas (Rich; Weaver, 2000) y, en España, prácticamente inexistentes (Lalueza; Girona, 2016). La bibliografía propone estudios en los que se afirma que no es posible evidenciar la influencia real de los think tanks en la formulación de políticas públicas, aunque es factible establecer la manera de ejercer dicha influencia, incluso determinar en qué momento de un determinado ciclo político despliegan mayor proyección (Abelson, 2009).

\section{Objetivos y metodología}

El objetivo de esta investigación es determinar la presencia de los principales think tanks españoles en medios de comunicación nacionales e internacionales, desde un punto de vista cuantitativo, considerando el período 2004-2018. Así mismo, se analiza la concentración de mensajes de dichos think tanks en atención al medio o agencia de medios.

Las variables utilizadas en esta investigación son el número de publicaciones de los think tanks españoles y su distribución en los medios nacionales e internacionales desde 2004 a 2018.

Durante los 15 años de análisis se distinguen tres etapas:

- Período A (2004-2007): se ha tomado como punto inicial el 1 de enero de 2004, momento en que se inicia la etapa final de la fase de crecimiento económico, previo al estallido de la burbuja inmobiliaria.

- Período B (2008-2012): los cinco años posteriores corresponden a la etapa de crisis, ajustes y reformas estructurales, incluyendo la reforma laboral (Ley 3/2012, de 6 de julio) (España, 2012a), así como el proceso de bancarización de las cajas de ahorro y la correspondiente solicitud de asistencia financiera (Memorando de entendimiento sobre condiciones de política sectorial financiera, de 10 de diciembre de 2012) (España, 2012b).

- Período C (2013-2018): los siguientes seis años corresponden a la fase de recuperación y crecimiento económico, finalizando el análisis el 31 de diciembre de 2018. Se incluye el seguimiento del desafío soberanista en Cataluña con las repercusiones políticas aparejadas y la moción de censura a Mariano Rajoy que convirtió a Pedro Sánchez en el séptimo presidente de la etapa democrática actual.

Para determinar los think tanks objeto de esta investigación se ha tomado como referencia inicial el ranking publicado por la University of Pennsylvania que, desde 2008 y de forma anual, categoriza este tipo de organizaciones. Para ello, se ha empleado el 2018 Global go to think tank index report (McGann, 2019) y se han incluido los 19 think tanks españoles que aparecen en el ranking, clasificados tanto por zona geográfica (Europa Occidental) como por áreas de interés (defensa y seguridad nacional, política local,

Según el Global go to think tank index report, en 2018 España tenía registrados 66 think tanks 
medioambiental, salud, desarrollo internacional, tecnología, seguridad alimentaria, entre otras). Por su parte, el trabajo de Tello-Benítez (2013) identifica 61 think tanks españoles, y se ha empleado para complementar las referencias de McGann (2019). Así, el corpus objeto de estudio queda configurado por 70 .

Diversos think tanks presentes en la base de datos no mostraban presencia en medios al inicio de la serie temporal analizada (2004) o habían desaparecido al finalizar dicha serie (2018), algunos como consecuencia de los episodios de corrupción política y económica protagonizados por los partidos que los habían impulsado. No obstante, su inclusión obedece a la representación mediática alcanzada por estos think tanks a lo largo de los 15 años analizados.

Para determinar la presencia de los think tanks en los medios se ha recurrido a Factiva ${ }^{\circledR}$, una base de datos de información perteneciente a Dow Jones \& Company que proporciona acceso a más de 35.000 fuentes de noticias de 200 países en 26 idiomas, destacando los diarios y revistas en papel y digitales, así como más de 400 agencias de noticias. En el caso de España, Factiva ${ }^{\circledR}$ permite el acceso online a 264 medios, incluyendo las agencias EFE y Europa Press.

Con el fin de objetivar el impacto de cada uno de los 70 think tanks analizados se ha seguido el siguiente protocolo de búsqueda en Factiva ${ }^{\circledR}$ :

1) Se buscó en título, resumen y noticia, utilizando el nombre completo del think tank con comillas, prescindiendo de sus acrónimos, a fin de evitar falsos positivos. Ello es debido a que los acrónimos de gran parte de los think tanks analizados representan otros términos identificativos de asociaciones empresariales, otro tipo de organizaciones o elementos incluidos aleatoriamente en las noticias, tanto en España como en el resto del mundo.

2) Para todos los medios de comunicación españoles, en cualquier idioma, para el período 2004-2018.

3) Se siguió el mismo criterio indicado en el paso (1), pero para todos los medios del mundo, excluidos los medios españoles.

Los resultados obtenidos en los pasos (2) y (3) permiten la calibración del grado de internacionalización del discurso logrado por cada uno de las organizaciones analizadas.

El análisis de la tendencia temporal para los 70 think tanks se ha llevado a cabo a través de una regresión exponencial cuya adherencia estadística se ajusta más adecuadamente a los datos disponibles.

Para la evaluación de la variabilidad de la presencia en medios a lo largo de la serie temporal se ha calculado la media de publicaciones para los 15 años analizados, su desviación estándar y el peso de esta desviación con respecto a la media.

\section{Resultados}

Como se ha señalado, el período analizado se extiende a lo largo de 15 años (2004-2018), correspondiendo los tres primeros (Período A: 20042007) con la fase final del ciclo de crecimiento económico previo a la crisis, los cinco siguientes incluyen la etapa de crisis, ajustes y reformas estructurales (Período B: 2008-2012), y los últimos seis (Período C: 2013-2018) de recuperación y crecimiento económico.

En la tabla 1 se muestran los 70 think tanks españoles objeto de estudio y el acrónimo elegido para referirse a ellos cuando no se dispone de uno propio. Se han ordenado de mayor a menor, en función del número total de publicaciones en las que han aparecido desde 2004 a 2018.

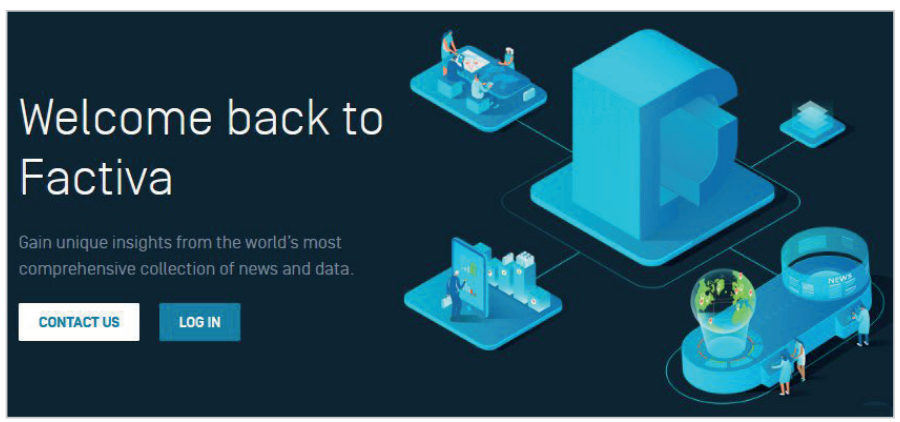

https://professional.dowjones.com/factiva 
Tabla 1. Think tanks españoles analizados para el período 2004-2018

\begin{tabular}{|c|c|c|}
\hline Think tank & Acrónimo/Abreviatura & N. noticias \\
\hline Fundación de Cajas de Ahorros & Funcas & 28.419 \\
\hline Círculo de Empresarios & CdEmp & 16.646 \\
\hline Fundación de Estudios de Economía Aplicada & Fedea & 13.582 \\
\hline Real Instituto Elcano & Elcano & 12.099 \\
\hline Instituto de Estudios Económicos & IEE & 11.795 \\
\hline Cercle d'Economia & CdEco & 8.909 \\
\hline Action Against Hunger Spain & Aahs & 8.487 \\
\hline Barcelona Centre for International Affairs & Cidob & 4.773 \\
\hline Fundación para el Análisis y los Estudios Sociales & Faes & 4.749 \\
\hline Fundación Ideas & Ideas & 4.594 \\
\hline Fundacion Alternativas & F.Alter & 4.590 \\
\hline Instituto de Estudios Fiscales & IEF & 4.321 \\
\hline Centre for Ecological Research and Forestry Applications & Creaf & 3.863 \\
\hline Barcelona Institute for Global Health & ISGlobal & 3.767 \\
\hline Club de Madrid & $C d M$ & 3.627 \\
\hline Fundación Carolina & F.Caro & 3.479 \\
\hline Fundación Pablo Iglesias & $F P I$ & 3.056 \\
\hline Institut Europeu de la Mediterrània & IEMed & 2.475 \\
\hline Sabino Arana Fundazioa & SAF & 2.332 \\
\hline Fundació Jaume Bofill & $F J B$ & 1.977 \\
\hline Fundación Ecología y Desarrollo & FED & 1.888 \\
\hline Civismo & CIV & 1.698 \\
\hline Instituto Español de Estudios Estratégicos & leee & 1.553 \\
\hline Centro de Estudios de la Defensa Nacional & Ceseden & 1.401 \\
\hline Fundació CatDem & Catdem & 1.270 \\
\hline Instituto Juan de Mariana & I.J.Mariana & 1.053 \\
\hline Fundación Independiente & F.Indep & 990 \\
\hline Instituto de Estudios sobre Conflictos y Acción Humanitaria & lecah & 738 \\
\hline Fundación Sistema & F.Sist & 692 \\
\hline Fundació Rafael Campalans & $F R C$ & 586 \\
\hline Fundación Internacional para la Libertad & FIL & 585 \\
\hline Fundación Economía Aragonesa & Fundear & 580 \\
\hline Centre d'Estudis Jordi Pujol & Cejp & 577 \\
\hline Fundación Galiza Sempre & FGS & 528 \\
\hline Institución Futuro & I.Futuro & 507 \\
\hline Gernika Gogoratuz. Centro de Investigación por la Paz & Gernika & 501 \\
\hline Instituto Franklin de Investigación en Estudios Norteamericanos & I.Franklin & 492 \\
\hline Fundación Cotec & Cotec & 473 \\
\hline Grupo de Estudios Estratégicos & Gees & 462 \\
\hline Fundació Josep Irla & $F J$ & 431 \\
\hline Fundación para las Relaciones Internacionales y Diálogo Exterior & Fride & 429 \\
\hline Fundación Encuentro & F.Encuentro & 401 \\
\hline Fundació Catalunya Oberta & F.C.Oberta & 390 \\
\hline Fundació Catalunya Europa & F.C.Europa & 348 \\
\hline Institute of Agrochemistry and Food Technology & laft & 338 \\
\hline Fundación Ciudadanía y Valores & Fcyv & 306 \\
\hline Fundación Europea Sociedad y Educación & Fesye & 286 \\
\hline Fundación Innovación Bankinter & $F I B$ & 267 \\
\hline Fundación Ortega y Gasset-Gregorio Marañón & F.Og-Gm & 264 \\
\hline
\end{tabular}




\begin{tabular}{|c|c|c|}
\hline Basque Centre for Climate Change & $B C 3$ & 226 \\
\hline Fundación de Investigaciones Marxistas & FIM & 219 \\
\hline Igadi. Instituto Galego de Análise e Documentación Internacional & Igadi & 202 \\
\hline Fundación Seminario de Investigación para la Paz & Fsip & 199 \\
\hline Cercle d'Estudis Sobiranistes & CES & 198 \\
\hline Centro de Toledo por la Paz & CITpax & 197 \\
\hline Institut d'Estudis Humanístics Miquel Coll i Alentorn & lehmca & 191 \\
\hline Aspen Institute España & AIE & 177 \\
\hline Euro-Mediterranean Study Commission & EuroMeSCo & 170 \\
\hline Fundació Ferrer i Guàrdia & FFG & 154 \\
\hline Centro Atlántico de Pensamiento Estratégico & Catpe & 148 \\
\hline Fundación Progreso y Democracia & Fpyd & 134 \\
\hline Fundación por la Europa de los Ciudadanos & FEC & 128 \\
\hline Instituto de Cuestiones Internacionales y Política Exterior & Incipe & 94 \\
\hline Fundació Nous Horitzons & FNH & 76 \\
\hline Centro de Desarrollo Internacional de la Universidad de Navarra & Ncid & 73 \\
\hline Fundació l'alternativa & $F L A$ & 37 \\
\hline Fundación Burke & Burke & 19 \\
\hline Genetic Resources Action International & Grain & 14 \\
\hline Red Iberoamericana de Estudios Internacionales & Ribei & 6 \\
\hline Persona + Democràcia Joaquim Xicoy & Pdjx & 2 \\
\hline
\end{tabular}

Fuente: Elaborado a partir de McGann (2019) y Tello-Benítez (2013)

A lo largo del período se observa una presencia mediática creciente $\left(R^{2}=0,9745\right)$ de los 70 think tanks, no quedando ligada a la evolución del ciclo económico ni a los cambios experimentados en la situación política nacional, que incluye cinco comicios y una moción de censura. Coincidiendo con el comienzo del proceso de recuperación económica iniciado en 2013, se constata un leve retroceso. No obstante, la representación mediática de los think tanks españoles recupera al año siguiente su senda de expansión (gráfico 1).

La presencia de los think tanks en la agenda mediática aumenta en el Período C (2013-2018), frente al Período A

(2004-2007), por lo que se observa una continuidad en la expansión de estas entidades dentro del debate público. El Período B (2008-2012) constituyó una palanca de crecimiento para su presencia en medios, contribuyendo de forma constante a su impulso y generalización.

Los datos obtenidos muestran que 16 think tanks $(22,8 \%)$ concentran el $80 \%$ de los contenidos generados en los 15 años analizados. De estos, los 6 de mayor presencia en medios explican el $50 \%$ de los contenidos asociados a los mismos (gráfico 2).
La presencia en medios de los think tanks es creciente a lo largo de los años, con independencia del ciclo económico $y$ de los cambios experimentados en la situación política nacional 


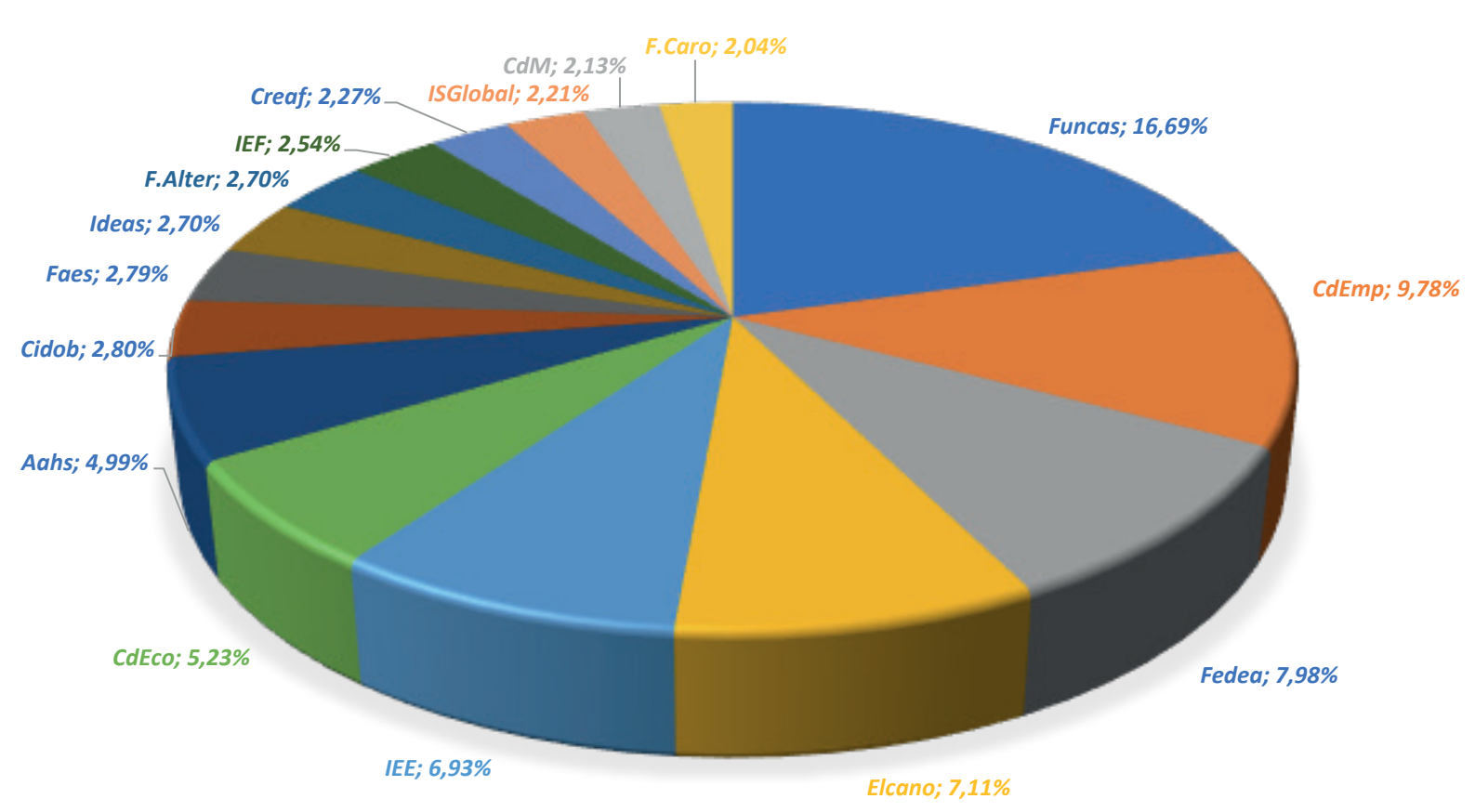

Gráfico 2. Importancia relativa de la presencia mediática de los think tanks españoles (2004-2018)

Tal y como se observa en la tabla 2, los seis think tanks con mayor presencia mediática concentran el 53,72\% de los contenidos generados entre 2004 y 2018. De estos, cinco son de carácter económico. Asimismo, se muestran los que están formalmente ligados a un partido político: Fundación Ideas y Fundación Alternativas se hallan vinculadas al Partido Socialista Obrero Español (PSOE), mientras que Fundación para el Análisis y los Estudios Sociales (FAES) está conectada con el Partido Popular (PP).

Tabla 2. Principales think tanks españoles que concentran el $80 \%$ de los contenidos

\begin{tabular}{|l|c|c|c|c|c|}
\hline \multicolumn{1}{|c|}{ Think tank } & Económico & Partido político & Media anual & Desv. est. & $\begin{array}{c}\text { Peso desv. est. } \\
\%\end{array}$ \\
\hline Funcas & Sí & No & $1.894,60$ & 754,61 & 39,83 \\
\hline CdEmp & Sí & No & $1.109,73$ & 604,34 & 54,46 \\
\hline Fedea & Sí & No & 905,47 & 738,93 & 81,61 \\
\hline Elcano & No & No & 806,60 & 466,87 & 57,88 \\
\hline IEE & Sí & No & 786,33 & 204,57 & 26,02 \\
\hline CdEco & Sí & No & 593,93 & 438,70 & 73,86 \\
\hline Aahs & No & No & 565,80 & 276,15 & 48,81 \\
\hline Cidob & No & So & 318,20 & 185,34 & 58,25 \\
\hline Faes & No & Sí & 316,60 & 125,71 & 39,70 \\
\hline Ideas & No & Sí & 306,27 & 332,48 & 108,56 \\
\hline F.Alter & No & No & 288,07 & 116,28 & 38,00 \\
\hline IEF & Sí & No & 257,53 & 79,52 & 27,60 \\
\hline Creaf & No & No & 251,13 & 426,65 & 63,99 \\
\hline ISGlobal & No & No & 241,80 & 151,33 & 169,89 \\
\hline CdM & No & No & 231,93 & 63,07 & 62,58 \\
\hline F.Caro & No & & 27,19 \\
\hline
\end{tabular}

Para los 15 años analizados, Funcas destaca con la mayor media anual. En cambio, la representación mediática de Fundación Carolina, registra una media nueve veces inferior a la de Funcas. 
Tabla 3. Contenidos publicados sobre think tanks en medios nacionales e internacionales

\begin{tabular}{|c|c|c|c|c|c|c|c|c|c|c|c|c|c|c|c|}
\hline TT/Año & 2004 & 2005 & 2006 & 2007 & 2008 & 2009 & 2010 & 2011 & 2012 & 2013 & 2014 & 2015 & 2016 & 2017 & 2018 \\
\hline Funcas & 818 & 963 & 732 & 1.003 & 1.739 & 1.857 & 2.209 & 2.520 & 1.826 & 1.526 & 2.408 & 2.317 & 2.918 & 2.643 & 2.940 \\
\hline CdEmp & 461 & 610 & 609 & 564 & 639 & 626 & 833 & 1.186 & 955 & 749 & 1.810 & 1.597 & 1.813 & 2.220 & 1.974 \\
\hline Fedea & 70 & 79 & 84 & 80 & 114 & 458 & 846 & 810 & 1.528 & 904 & 1.380 & 1.447 & 1.830 & 2.062 & 1.890 \\
\hline Elcano & 392 & 533 & 423 & 557 & 431 & 409 & 467 & 527 & 688 & 830 & 939 & 1.041 & 1.495 & 1.727 & 1.640 \\
\hline IEE & 445 & 520 & 438 & 561 & 864 & 788 & 793 & 939 & 958 & 1.002 & 890 & 730 & 1.074 & 899 & 894 \\
\hline CdEco & 92 & 92 & 136 & 240 & 347 & 244 & 370 & 440 & 531 & 797 & 1.006 & 1.323 & 1.010 & 1.342 & 939 \\
\hline Aahs & 301 & 331 & 317 & 340 & 374 & 391 & 421 & 467 & 450 & 592 & 809 & 696 & 824 & 962 & 1.212 \\
\hline Cidob & 193 & 170 & 230 & 143 & 174 & 149 & 162 & 255 & 353 & 282 & 349 & 502 & 430 & 689 & 692 \\
\hline Faes & 273 & 517 & 344 & 484 & 368 & 471 & 409 & 430 & 242 & 301 & 223 & 218 & 136 & 150 & 183 \\
\hline Ideas & 10 & 41 & 30 & 24 & 120 & 482 & 603 & 939 & 541 & 991 & 233 & 103 & 96 & 53 & 328 \\
\hline F.Alter & 171 & 266 & 249 & 265 & 309 & 487 & 308 & 217 & 211 & 213 & 365 & 329 & 206 & 399 & 595 \\
\hline IEF & 199 & 349 & 281 & 273 & 284 & 216 & 313 & 227 & 206 & 307 & 492 & 216 & 321 & 247 & 390 \\
\hline Creaf & 89 & 168 & 235 & 209 & 64 & 90 & 110 & 207 & 214 & 200 & 294 & 383 & 546 & 513 & 541 \\
\hline ISGlobal & 0 & 1 & 0 & 0 & 0 & 3 & 88 & 34 & 98 & 90 & 263 & 269 & 484 & 971 & 1.466 \\
\hline$C d M$ & 145 & 673 & 279 & 295 & 147 & 222 & 143 & 127 & 177 & 172 & 179 & 496 & 231 & 191 & 150 \\
\hline F.Caro & 199 & 207 & 324 & 248 & 264 & 229 & 249 & 225 & 316 & 175 & 145 & 209 & 146 & 185 & 358 \\
\hline
\end{tabular}

Fuente: elaborado a partir de datos de Factiva ${ }^{\circledR}$

Siguiendo el análisis de la serie temporal (tabla 3), Fedea experimenta una notable evolución, pasando de estar presente en 70 publicaciones en 2004 a 1.980 en 2018.

Se observa que Funcas y Círculo de Empresarios experimentan un retroceso de su presencia mediática con el comienzo de la recuperación (2013), si bien impulsan su tendencia al alza a partir de 2014, afianzando su presencia en la fase de crecimiento, concretamente entre 2016 y 2018.

En cambio, Fedea intensifica su presencia en medios durante 2012 y se reduce significativamente (-41\%) en 2013. No obstante, en 2014 regresa a la tendencia interanual de crecimiento que le lleva a convertirse, en 2018, en el segundo think tank español con mayor nivel de representación mediática.

Durante el Período B (2008-2012), el gobierno español decidió diseñar e impulsar políticas públicas fundamentadas en ajustes y reformas estructurales, como respuesta al problema de déficit y deuda sufridos por la economía española en el contexto de la crisis del euro. Este aspecto se encuentra alineado con estudios recientes en los que se constata el papel esencial de estas organizaciones para la creación de un consenso dirigido a favorecer la reestructuración del sistema financiero y la creación de narrativas alineadas con las políticas de austeridad (Parrilla; Almiron; Xifra, 2016).

Diversas investigaciones sugieren que los think tanks tienen una influencia potencial en el clima de opinión, especialmente en las etapas iniciales de desarrollo de las políticas públicas (Castaño; Méndez; Galindo, 2015; Rich, 2004), puesto que suministran información relevante para asesorar a los tomadores de decisiones de los gobiernos (Misztal, 2012; Denham, 2005). Por el contrario, la evidencia obtenida sobre Fedea y Cercle d'Economia, dos de los think tanks que registraron un mayor crecimiento de su presencia en medios, muestra un incremento continuo, comportamiento alineado con otros estudios que destacan la función de la cobertura mediática a lo largo de todo el ciclo económico (Soroka et al., 2013).

Más allá de los think tanks de carácter económico, merece especial atención ISGlobal, orientado a temas de salud pública que, en apenas una década, ha experimentado una evolución considerable en cuanto a presencia en medios, pasando de tener 3 referencias en 2009, a registrar 1.466 en 2018.

Sin embargo, tal y como se observa en la tabla 4 , los think tanks cuya temática es fundamentalmente económica (en adelante, económicos), concentran un 63,40\% del total de contenidos. Las publicaciones aparecidas en medios con referencia a los no exclusivamente económicos (en adelante, no económicos) representan sólo uno de cada tres contenidos (36,60\% del total).
Desde el inicio de la crisis, los think tanks de carácter económico alcanzaron un mayor nivel de presencia en medios, tanto nacionales como internacionales 
Tabla 4. Distribución de publicaciones entre think tanks (TT) económicos y no económicos (2004-2018)

\begin{tabular}{|c|c|c|c|}
\hline Años & Publicaciones TT económicos & $\begin{array}{c}\text { Publicaciones TT } \\
\text { no económicos }\end{array}$ & $\begin{array}{c}\text { Económicos sobre total } \\
\text { \% }\end{array}$ \\
\hline 2004 & 2.230 & 1.628 & 57,80 \\
\hline 2005 & 3.286 & 2.234 & 59,53 \\
\hline 2006 & 2.559 & 2.152 & 54,32 \\
\hline 2007 & 3.016 & 2.270 & 57,06 \\
\hline 2008 & 4.134 & 2.104 & 66,27 \\
\hline 2009 & 4.411 & 2.711 & 61,93 \\
\hline 2010 & 5.507 & 2.817 & 66,16 \\
\hline 2011 & 6.249 & 3.301 & 65,43 \\
\hline 2012 & 6.181 & 3.113 & 66,51 \\
\hline 2013 & 5.457 & 3.674 & 59,76 \\
\hline 2014 & 8.165 & 3.620 & 69,28 \\
\hline 2015 & 8.126 & 3.750 & 68,42 \\
\hline 2016 & 9.197 & 4.363 & 67,82 \\
\hline 2017 & 9.604 & 5.649 & 62,96 \\
\hline 2018 & 9.177 & 7.015 & 56,68 \\
\hline
\end{tabular}

Fuente: elaborado a partir de datos de Factiva $^{\circledR}$

La tendencia de la presencia en medios de think tanks económicos y no económicos es creciente a lo largo de los 15 años analizados, aumentando cada vez más la brecha existente de la representación mediática entre ambos tipos. Desde el inicio de la crisis, los económicos alcanzaron un mayor nivel de presencia en medios, tanto nacionales como internacionales (gráfico 3).

Tras la consolidación de la fase de recuperación (Período C: 2013-2018) y, más específicamente a partir de 2015, se percibe un claro incremento de la presencia en medios de los think tanks no económicos, reduciendo la diferencia con respecto a los económicos.

Por otra parte, al examinar los tres think tanks relacionados directamente con un partido político (Fundación Ideas, Fundación Alternativas y Faes), se constata que su comportamiento a lo largo del tiempo es estable con pequeñas oscilaciones. La tabla 5 muestra la presencia en medios de los think tanks ligados a partidos políticos, representando entre un $11,77 \%$ y un $14,62 \%$ del total para el período previo a la crisis (Período A: 2004-2007).

Durante la etapa de crisis, reformas y ajustes (Período B: 2008-2012), su impacto aumentó hasta alcanzar el $20,22 \%$ de presencia en medios. Sin embargo, en el momento de mayor profundidad de la crisis, iniciaron un descenso en su capacidad de impacto en la agenda pública, cayendo al 3,23\%. En este sentido, los think tanks pierden influencia en medios cuando estos perciben que existe una clara vinculación con un partido político (Lalueza; Girona, 2016).

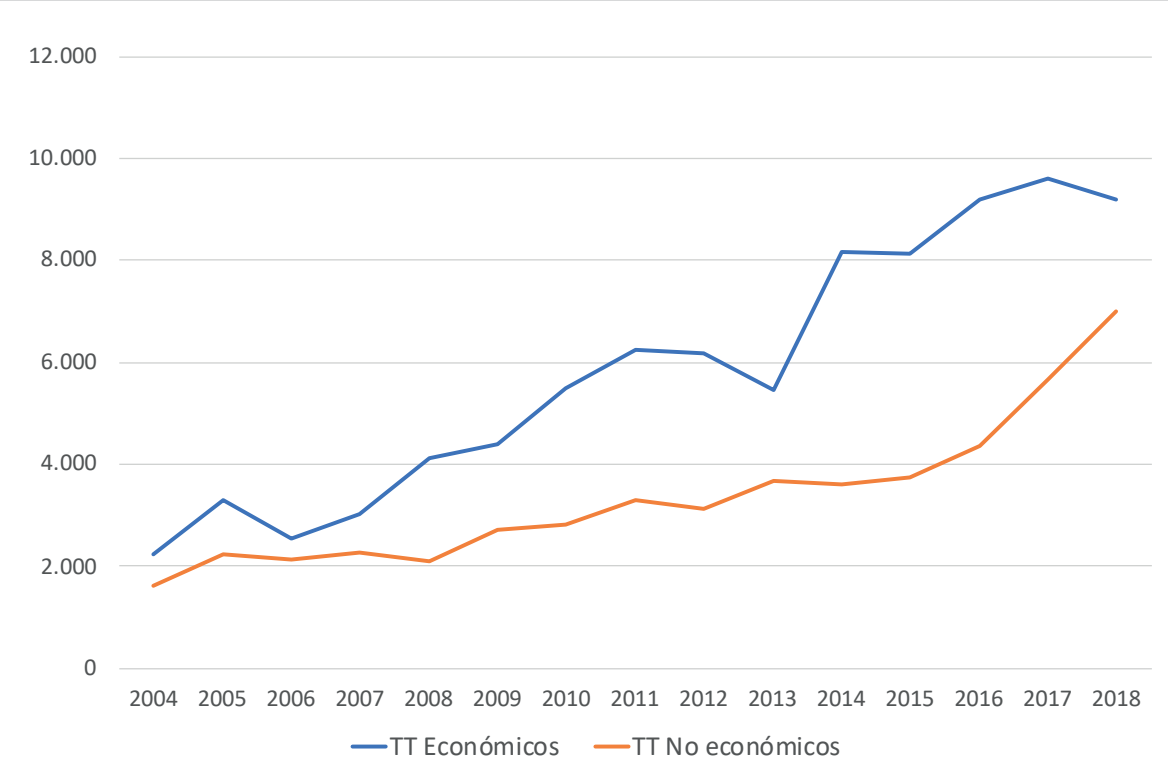

Gráfico 3. Comportamiento longitudinal de la presencia mediática de los think tanks económicos frente a los no económicos

Fuente: elaborado a partir de datos de Factiva ${ }^{\circledR}$. 
Tabla 5. Distribución de publicaciones entre think tanks ligados y no ligados a partidos políticos (2004-2018)

\begin{tabular}{|c|c|c|c|}
\hline Años & Ligados a partidos & No ligados a partidos & Ligados a partidos sobre el total \\
\hline 2004 & 454 & 3.404 & 11,77 \\
\hline 2005 & 824 & 4.696 & 14,93 \\
\hline 2006 & 623 & 4.088 & 13,22 \\
\hline 2007 & 773 & 4.513 & 14,62 \\
\hline 2008 & 797 & 5.441 & 12,78 \\
\hline 2009 & 1.440 & 5.682 & 20,22 \\
\hline 2010 & 1.320 & 7.004 & 15,86 \\
\hline 2011 & 1.586 & 7.964 & 16,61 \\
\hline 2012 & 994 & 8.300 & 10,70 \\
\hline 2013 & 1.505 & 7.626 & 16,48 \\
\hline 2014 & 821 & 10.964 & 6,97 \\
\hline 2015 & 650 & 11.226 & 5,47 \\
\hline 2016 & 438 & 13.122 & 3,23 \\
\hline 2017 & 602 & 14.651 & 3,95 \\
\hline 2018 & 1.106 & 15.086 & 6,83 \\
\hline Total & 13.933 & 123.767 & \\
\hline Importancia & $10,12 \%$ & $89,88 \%$ & \\
\hline
\end{tabular}

Fuente: elaborado a partir de datos de Factiva $^{\circledR}$

Por otra parte, aquellos que no presentan relación con partidos políticos muestran una evolución radicalmente opuesta, con un incremento exponencial, especialmente tras el inicio de la recuperación económica, a partir del cuarto trimestre de 2013. En el gráfico 4 se representa la evolución dinámica de la presencia en medios de los think tanks en función de su vinculación con partidos políticos.

El análisis de los medios y agencias que hacen referencia a los think tanks españoles que concentran el $80 \%$ de los contenidos, devuelve una presencia en 257 medios procedentes de 44 países. Al

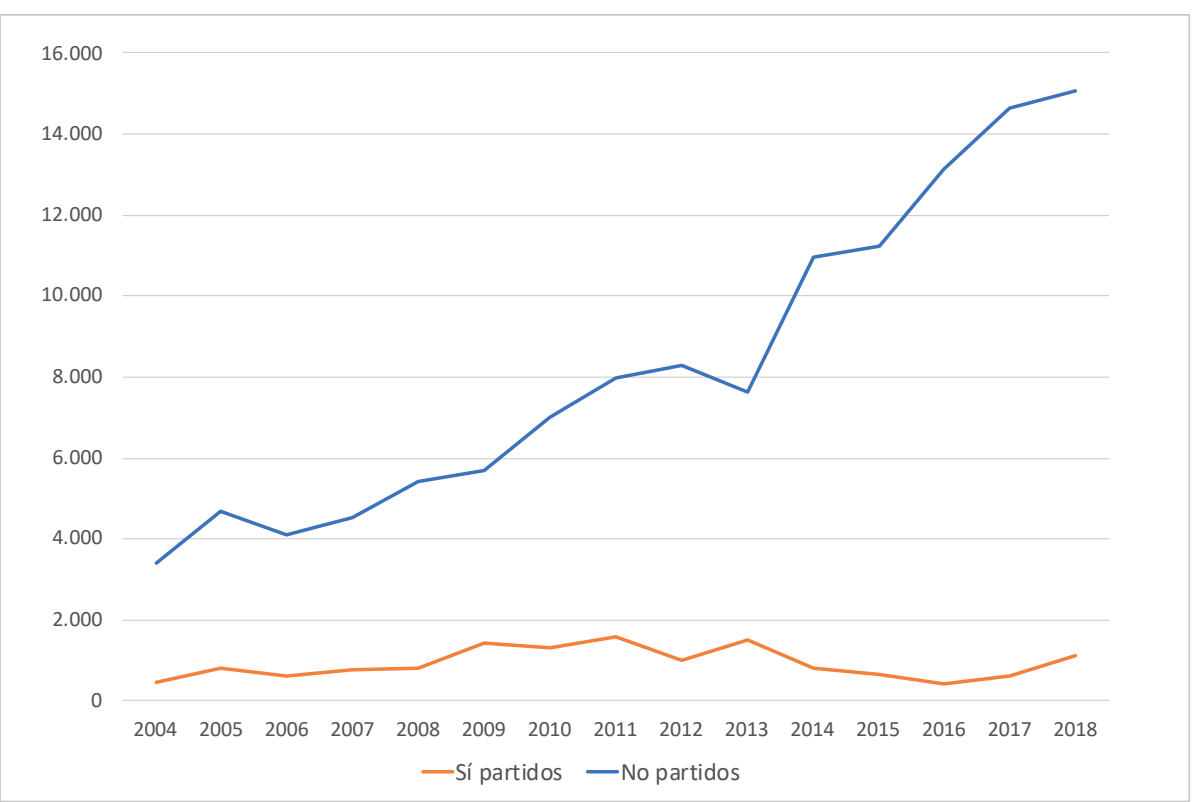

Gráfico 4. Comportamiento longitudinal de los think tanks ligados y no ligados a partidos políticos Fuente: elaborado a partir de datos de Factiva $^{\circledR}$ margen de España, los países con mayor número de medios que incorporaron referencias a los think tanks españoles fueron: Estados Unidos, Reino Unido, Italia, México, Francia, Argentina, India, China, Colombia, Chile, Rusia, Alemania y Portugal (tabla 6).

El análisis del grado de internacionalización de los think tanks españoles ofrece tasas destacadas para Creaf, Club de Madrid, Cidob, Fundación Carolina y Real Instituto Elcano, todos ellos no económicos. Para los 15 años analizados, de cada 10 noticias que hacen referencia a Creaf, think tank de temática medioambiental, cuatro aparecen en medios internacionales.

Por su parte, los think tanks económicos evidencian una capacidad limitada en la internacionalización de su proyección. De hecho, Instituto de Estudios Fiscales, con un 6,60\%, es el think tank económico con mayor tasa de internacionalización en medios. Entre los think tanks políticos, una de cada 10 referencias a Fundación Ideas, fueron publicadas en medios internacionales, duplicando la tasa de internacionalización de FAES, y multiplicando por cinco la tasa de Fundación Alternativas (tabla 7). 
Tabla 6. Distribución de los medios internacionales por países

\begin{tabular}{|c|c|c|c|}
\hline País & Medios & País & Medios \\
\hline Alemania & 4 & Italia & 12 \\
\hline Argentina & 7 & Jordania & 1 \\
\hline Australia & 3 & Kazajistán & 1 \\
\hline Azerbaiyán & 3 & Luxemburgo & 1 \\
\hline Bolivia & 1 & México & 9 \\
\hline Brasil & 3 & Noruega & 1 \\
\hline Bulgaria & 1 & Panamá & 1 \\
\hline Canadá & 3 & Paraguay & 1 \\
\hline Chile & 6 & Perú & 2 \\
\hline China & 6 & Portugal & 4 \\
\hline Colombia & 6 & Reino Unido & 12 \\
\hline Costa Rica & 1 & República Checa & 2 \\
\hline Dubái & 1 & Rusia & 5 \\
\hline Ecuador & 1 & Suecia & 1 \\
\hline EUA & 23 & Suiza & 2 \\
\hline España & 107 & Tailandia & 1 \\
\hline Europa & 1 & Taiwán & 1 \\
\hline Francia & 8 & Túnez & 1 \\
\hline Guatemala & 1 & Uruguay & 1 \\
\hline India & 6 & Venezuela & 2 \\
\hline Irán & 1 & Sudáfrica & 1 \\
\hline Irlanda & 1 & Estonia & 1 \\
\hline
\end{tabular}

Tabla 7. Grado de internacionalización de los 16 think tanks que concentran el $80 \%$ de los contenidos

\begin{tabular}{|l|c|c|}
\hline Think tank & $\begin{array}{c}\text { Publicaciones } \\
\text { nacionales } \\
\%\end{array}$ & $\begin{array}{c}\text { Publicaciones } \\
\text { internacionales } \\
\%\end{array}$ \\
\hline Creaf & 57,18 & 42,82 \\
\hline CdM & 67,88 & 32,12 \\
\hline Cidob & 79,98 & 20,02 \\
\hline F.Caro & 86,62 & 13,38 \\
\hline ISGlobal & 88,14 & 11,86 \\
\hline Ideas & 88,36 & 11,64 \\
\hline Elcano & 90,10 & 9,90 \\
\hline IEE & 92,85 & 7,15 \\
\hline IEF & 93,40 & 6,60 \\
\hline Fedea & 94,60 & 5,40 \\
\hline Faes & 94,60 & 5,40 \\
\hline Aahs & 94,81 & 5,19 \\
\hline Funcas & 96,85 & 3,15 \\
\hline F.Alter & 97,93 & 2,07 \\
\hline CdEmp & 98,03 & 1,97 \\
\hline CdEco & 98,59 & \\
\hline
\end{tabular}

Fuente: Elaborado a partir de datos de Factiva ${ }^{\circledR}$

Fuente: elaborado a partir de datos de Factiva ${ }^{\circledR}$

A fin de facilitar el análisis y comprensión de la información disponible, la concentración de los contenidos de think tanks en medios y agencias ha sido tabulada por horquillas. Las tablas 8 y 9 muestran los índices de concentración de contenidos en un medio o agencia, cuando estos son superiores al $1 \%$ sobre el total de publicaciones registradas para cada think tank para un medio durante los 15 años objeto de estudio.

Los think tanks han sido agrupados según sean económicos o no económicos, y los medios y agencias según sean nacionales o internacionales.

Europa Press y EFE aglutinan una gran parte de los contenidos, tanto para los think tanks económicos como para los no económicos.

Círculo de Empresarios, Club de Madrid y Cidob concentran su penetración en la agenda mediática a partir de unos pocos medios que cuentan con una gran representatividad. En el caso de Club de Madrid, los medios en los que tiene mayor presencia son El país, $A B C$, El periódico y El mundo, seguidos por La vanguardia y el Diario montañés.

En base a su proyección autonómica, Cercle d'Economia y Cidob concentran gran parte de su discurso mediático, además, en torno a El periódico y La vanguardia. Asimismo, Cidob cuenta con una penetración importante en El país y Expansión, seguidos por $A B C$ y $E I$ mundo.

Dentro del análisis de los think tanks ligados a partidos políticos, Fundación Alternativas encuentra un mayor índice de concentración en El país, al mismo tiempo que cuenta con una dispersión en medios superior que Faes y Fundación Ideas. Fundación Alternativas disemina su presencia en medios regionales en los que la penetración de Faes y Fundación Ideas es testimonial o inexistente. Es el caso de La voz de Galicia, Las provincias o La Rioja.

En un análisis de los medios de prensa salmón para los think tanks de carácter económico, observamos que Expansión es el único que ofrece soporte significativo a los contenidos de Fedea. Este medio, junto a Cinco días y El economista, representan concentraciones relevantes de los contenidos de Funcas, Círculo de Empresarios, Instituto de Estudios Económicos e Instituto de Estudios Fiscales.

En el análisis de la presencia internacional en medios destaca el papel de la agencia France Presse para los contenidos de Club de Madrid, Real Instituto Elcano y Cidob, o la agencia Notimex para Club de Madrid, Fundación Carolina y, con un menor impacto, Real Instituto Elcano y Faes. Fundación Ideas encuentra el respaldo internacional de sus contenidos en Colombia a través de El tiempo. Por último, algunos think tanks cuya penetración internacional en prensa es dispersa y poco concentrada son Círculo de Empresarios, Cercle d'Economia, Instituto de Estudios Fiscales, Fundación Alternativas e ISGlobal. 
Tabla 8. Presencia de los think tanks económicos en medios nacionales e internacionales (2004-2018)

\begin{tabular}{|c|c|c|c|c|c|c|}
\hline & Fedea & Funcas & CdEmp & CdEco & IEE & IEF \\
\hline \multicolumn{7}{|l|}{ Nacionales } \\
\hline Europa Press & $\&$ & $\mathrm{O}$ & $\square$ & $\square$ & $\square$ & $\square$ \\
\hline Agencia EFE & $\square$ & $\mathrm{O}$ & $\mathrm{O}$ & $\square$ & $\square$ & $\mathrm{O}$ \\
\hline El país & $\mathrm{O}$ & $\mathrm{O}$ & 0 & & 0 & $\mathrm{O}$ \\
\hline$A B C$ & $\mathrm{O}$ & $\mathrm{O}$ & $\mathrm{O}$ & & 0 & 0 \\
\hline El periódico & $\mathrm{O}$ & $\mathrm{O}$ & $\mathrm{O}$ & $\&$ & $\mathrm{O}$ & 0 \\
\hline El mundo & $\mathrm{O}$ & 0 & $\mathrm{O}$ & $x$ & $\mathrm{O}$ & 0 \\
\hline Expansión & $x$ & $\mathrm{O}$ & $\mathrm{O}$ & & $\mathrm{O}$ & 0 \\
\hline La opinión & $\mathrm{O}$ & $\mathrm{O}$ & $\mathrm{O}$ & & $\mathrm{O}$ & 0 \\
\hline La vanguardia & $x$ & $\mathrm{O}$ & $x$ & $\square$ & $x$ & 0 \\
\hline Lavozdigital.es & & & $x$ & & & \\
\hline Lagaceta & $x$ & $\mathrm{O}$ & $x$ & & 0 & 0 \\
\hline La verdad & $x$ & $x$ & $x$ & & $\mathrm{O}$ & 0 \\
\hline La Rioja & & $x$ & & & & $x$ \\
\hline El norte de Castilla & $x$ & $x$ & $x$ & & & $x$ \\
\hline Diario montañés & $x$ & $x$ & & & & $x$ \\
\hline El correo & $x$ & $x$ & $\mathrm{O}$ & & & \\
\hline Sur & $x$ & $x$ & $x$ & & & $x$ \\
\hline Las provincias & & & $\mathrm{O}$ & & $\mathrm{O}$ & \\
\hline Cinco días & & 0 & 0 & & 0 & 0 \\
\hline El diario de León & $x$ & $x$ & $x$ & & & $x$ \\
\hline La voz de Galicia & & $x$ & $\mathrm{O}$ & & $x$ & $x$ \\
\hline Hoy & $x$ & $x$ & & & & $x$ \\
\hline La nueva España & & & & & & $x$ \\
\hline \multicolumn{7}{|l|}{ Ideal } \\
\hline El diario vasco & & & $x$ & & & \\
\hline El comercio & & $x$ & & & & \\
\hline Eleconomista & & $\mathrm{O}$ & $x$ & & $\mathrm{O}$ & 0 \\
\hline \multicolumn{7}{|l|}{ Internacionales } \\
\hline \multicolumn{7}{|c|}{ Agence France Presse (Francia) } \\
\hline Notimex (México) & $x$ & & & & & \\
\hline \multicolumn{7}{|c|}{ Financial Times (Reino Unido) } \\
\hline Reuters (Reino Unido) & & $x$ & & & $x$ & \\
\hline \multicolumn{7}{|c|}{ The Associated Press (EUA) } \\
\hline \multicolumn{7}{|c|}{ Dow Jones Newswires (EUA) } \\
\hline La nación (Argentina) & & & & & $x$ & \\
\hline \multicolumn{7}{|l|}{ Ansa (Italia) } \\
\hline \multicolumn{7}{|l|}{ El mercurio (Chile) } \\
\hline \multicolumn{7}{|l|}{ El tiempo (Colombia) } \\
\hline \multicolumn{7}{|l|}{ Reforma (México) } \\
\hline La razón (Bolivia) & & & & & & \\
\hline
\end{tabular}

\& Medio que recoge entre el $20,01 \%$ y el $30 \%$ de las noticias del think tank para el período 2004-2018

$\square \quad$ Medio que recoge entre el $10,01 \%$ y el $20 \%$ de las noticias del think tank para el período 2004-2018

O Medio que recoge entre el 2,01\% y el $10 \%$ de las noticias del think tank para el período 2004-2018

$X \quad$ Medio que recoge entre el $1 \%$ y el $2 \%$ de las noticias del think tank para el período 2004-2018 
Tabla 9. Presencia de los think tanks no económicos en medios nacionales e internacionales (2004-2018)

\begin{tabular}{|c|c|c|c|c|c|c|c|c|c|c|}
\hline & Elcano & Cidob & F.Alter & Faes & Ideas & $C d M$ & ISGlobal & F.Caro & Aahs & Creaf \\
\hline \multicolumn{11}{|l|}{ Nacionales } \\
\hline Europa Press & $\square$ & $\square$ & $\square$ & $\&$ & $\&$ & $\&$ & $\square$ & $*$ & $*$ & $\square$ \\
\hline Agencia EFE & $\mathrm{O}$ & $\mathrm{O}$ & $\mathrm{O}$ & $\square$ & $\mathrm{O}$ & $\square$ & $\square$ & $\square$ & $\mathrm{O}$ & $\square$ \\
\hline Elpais & $\mathrm{O}$ & $\mathrm{O}$ & $\square$ & $\mathrm{O}$ & $\mathrm{O}$ & $\mathrm{O}$ & $\mathrm{O}$ & $\mathrm{O}$ & $\mathrm{O}$ & \\
\hline$A B C$ & $\mathrm{O}$ & $x$ & $\mathrm{O}$ & $\mathrm{O}$ & $\mathrm{O}$ & $\mathrm{O}$ & $\mathrm{O}$ & $\mathrm{O}$ & $\mathrm{O}$ & $x$ \\
\hline El periódico & $\mathrm{O}$ & $\square$ & $\mathrm{O}$ & $\mathrm{O}$ & $\mathrm{O}$ & $\mathrm{O}$ & $\mathrm{O}$ & $\mathrm{O}$ & $\mathrm{O}$ & $\mathrm{O}$ \\
\hline El mundo & $\mathrm{O}$ & $x$ & $\mathrm{O}$ & $\mathrm{O}$ & $\mathrm{O}$ & $\mathrm{O}$ & $\mathrm{O}$ & $\mathrm{O}$ & $\mathrm{O}$ & \\
\hline Expansión & $\mathrm{O}$ & $\mathrm{O}$ & $\mathrm{O}$ & $x$ & & & & $x$ & & \\
\hline La opinión & $\mathrm{O}$ & & $x$ & $\mathrm{O}$ & $\mathrm{O}$ & & $x$ & $\mathrm{O}$ & $x$ & \\
\hline La vanguardia & $\mathrm{O}$ & $\square$ & $x$ & $x$ & $x$ & $x$ & $\mathrm{O}$ & & $x$ & $\mathrm{O}$ \\
\hline Lavozdigital.es & $x$ & & & & & & $x$ & & & $x$ \\
\hline Lagaceta & $x$ & & $\mathrm{O}$ & $x$ & $\mathrm{O}$ & & & & & \\
\hline La verdad & $x$ & & $x$ & $x$ & $x$ & & & & & \\
\hline LaRioja & $x$ & & $x$ & & & & $x$ & & $x$ & \\
\hline El norte de Castilla & $x$ & & $x$ & $x$ & $x$ & & & & & \\
\hline Diario montañés & $x$ & & $\mathrm{O}$ & $x$ & & $x$ & & $\mathrm{O}$ & & \\
\hline El correo & $x$ & & $x$ & $x$ & & & & & $x$ & \\
\hline Sur & $x$ & & $x$ & $x$ & & & & & & \\
\hline Las provincias & $x$ & & $x$ & & & & & & $x$ & \\
\hline Cinco días & $x$ & & $\mathrm{O}$ & & $x$ & & & $x$ & & \\
\hline El diario de León & $x$ & & $x$ & $x$ & $x$ & & & $\mathrm{O}$ & & \\
\hline La voz de Galicia & $x$ & & $x$ & & & & & & $\mathrm{O}$ & \\
\hline Hoy & $x$ & & $x$ & $x$ & $x$ & & & & $x$ & \\
\hline La nueva España & $x$ & & & & $x$ & & & & & \\
\hline Ideal & $x$ & & & & & & $x$ & & & \\
\hline El diario vasco & & & $x$ & & & & & & & \\
\hline El comercio & & & $x$ & & & & & & & \\
\hline El economista & & & & & & & & $x$ & & \\
\hline \multicolumn{11}{|l|}{ Internacionales } \\
\hline Agence France Presse (Francia) & $\mathrm{O}$ & $\mathrm{O}$ & & & & $\mathrm{O}$ & & & & \\
\hline Notimex (México) & $x$ & & & $x$ & & $\mathrm{O}$ & & $\mathrm{O}$ & $\mathrm{O}$ & \\
\hline Financial Times (Reino Unido) & $x$ & $x$ & & & & & & & & \\
\hline Reuters (Reino Unido) & & & & & & & & & & $x$ \\
\hline The Associated Press (EUA) & & & & & & $x$ & & & & \\
\hline Dow Jones Newswires (EUA) & & & & & & & & & & $\mathrm{O}$ \\
\hline La nación (Argentina) & & & & & & & & $x$ & $x$ & \\
\hline Ansa (Italia) & & & & & & & & & & $x$ \\
\hline El mercurio (Chile) & & & & & & $x$ & & & & \\
\hline El tiempo (Colombia) & & & & & $\mathrm{O}$ & & & & & \\
\hline Reforma (México) & & & & & & & & $x$ & $x$ & \\
\hline La razón (Bolivia) & & $\mathrm{O}$ & & & & & & & & \\
\hline
\end{tabular}

* $\quad$ Medio que recoge más del 30\% de las noticias del think tank para el período 2004-2018

\& $\quad$ Medio que recoge entre el 20,01\% y el 30\% de las noticias del think tank para el período 2004-2018

Medio que recoge entre el 10,01\% y el 20\% de las noticias del think tank para el período 2004-2018

Medio que recoge entre el 2,01\% y el 10\% de las noticias del think tank para el período 2004-2018

$\mathrm{X} \quad$ Medio que recoge entre el 1\% y el 2\% de las noticias del think tank para el período 2004-2018 


\section{Conclusiones}

La presencia mediática de los think tanks ha sido creciente a lo largo de los años, con independencia del ciclo económico y de los cambios en la situación política nacional. Este aspecto se alinea con McGann (2019), que destaca el incremento del alcance e impacto del trabajo de estas organizaciones.
Se constata que los think tanks españoles muestran un gran índice de concentración. La presencia en medios de 16 de ellos explica el $80 \%$ del discurso total de los 70 analizados

Se constata que los think tanks españoles muestran un gran índice de concentración. La presencia en medios de 16 de ellos explica el $80 \%$ del discurso total de los 70 analizados. Los seis con mayor impacto mediático representan uno de cada dos contenidos publicados.

La concentración del discurso de tales instituciones en medios se incrementa a lo largo del período estudiado, no mostrando correlaciones relevantes con respecto al momento del ciclo económico o el signo electoral que detenta el gobierno. Sin embargo, en un análisis de detalle, los ligados a partidos políticos muestran una tendencia estable, que no se ve aumentada en la serie temporal. Estos resultados coinciden con los estudios de Lalueza y Girona (2016) en los que se confirma que la presencia en medios de los think tanks vinculados a partidos políticos es menor que la de los que se presentan a la opinión pública como independientes.

A pesar de ello, mantienen su representación mediática. Este aspecto podría explicarse por el fuerte arraigo que, históricamente, han tenido los think tanks que nacieron bajo el auspicio de los partidos políticos, y el grado de afinidad ideológica con las líneas editoriales de los diferentes medios de comunicación (Rich; Weaver, 2000), o la estrategia de convertirse en fuente de referencia para determinados temas (Lalueza; Girona, 2016). En cualquier caso, los think tanks españoles están lejos del grado de influencia que este tipo de organizaciones ejerce en los países de cultura anglosajona (Ponsa-Herrera; González-Capitel, 2015).

Asimismo, es destacable que la presencia de los think tanks dependientes de los partidos no acompaña la tendencia creciente del resto de think tanks. Por tanto, cabría examinar si esta circunstancia también está relacionada con la crisis de legitimidad de los partidos políticos españoles y en qué medida. Los agentes políticos son incapaces de frenar los numerosos casos de corrupción y existe malestar debido a que el sistema de representación democrática

"arrastra deficiencias desde su instauración en el proceso de transición a la democracia después de casi 40 años de dictadura. A este déficit hay que sumar las desviaciones y los vicios generados por más de 30 años de funcionamiento corporativista de los partidos mayoritarios" (Tejerina-Montaña; Perugorría, 2017).

En una evaluación del grado de concentración de su discurso en medios, cinco think tanks de carácter económico (Funcas, Círculo de Empresarios, Fedea, Instituto de Estudios Económicos y Cercle d'Economia) se vuelven progresivamente hegemónicos, llegando a representar uno de cada dos contenidos de los 70 analizados.

Pese a la importancia internacional de la crisis económica española, los think tanks económicos apenas lograron índices de internacionalización relevantes en sus contenidos. Así, su capacidad de influencia internacional se muestra muy limitada en el contexto de la crisis financiera global, aun cuando el caso español representaba un ejemplo y referencia para el diseño de políticas públicas de ajuste y austeridad en otros países en crisis de la zona euro. Por el contrario, los think tanks que logran incrementar el efecto spillover internacional de sus mensajes son de carácter no económico, destacando Creaf, de temática medioambiental.

Los think tanks aparecen en la esfera mediática a partir de la producción de investigaciones. De hecho, una de las principales funciones de estas entidades es difundir sus hallazgos e ideas en medios para influir en los debates públicos. Estudios posteriores deberían examinar si la concentración en medios de unos pocos think tanks, puede llegar a empobrecer el debate público y reducir la pluralidad de ideas. Del mismo modo, próximas investigaciones deben centrarse en evaluar cómo la financiación de los think tanks puede condicionar las opiniones que proyectan en los medios (Arshed, 2017).

\section{Referencias}

Abelson, Donald E. (2009). Do think tanks matter?: Assessing the impact of public policy institutes. Montreal: McGiII-Queen's University Press. ISBN: 9780773523173

Abelson, Donald E. (2012). "Think-tanks, social democracy and social policy". Parliamentary affairs, v. 66, n. 4, pp. $894-902$. https://doi.org/10.1093/pa/gss051

Arshed, Norin (2017). "The origins of policy ideas: The importance of think tanks in the enterprise policy process in the UK". Journal of business research, v. 71, pp. 74-83.

https://doi.org/10.1016/j.jbusres.2016.10.015
Los think tanks que logran incrementar el efecto spillover internacional de sus mensajes son de temática diversa, que no fundamentan su carácter sobre temas exclusivamente económicos 
Baier, Gerald; Bakvis, Herman (2001). "Think tanks and political parties: competitors or collaborators?". Isuma: Canadian journal of policy research, v. 2, n. 1, pp. 107-113.

Barberà, Pablo; Arregui, Javier (2011). Naturaleza e influencia de los think tanks en el proceso politico en España (working paper n. 292). Barcelona: Institut de Ciències Polítiques i Socials. Universitat Autònoma de Barcelona.

https://www.icps.cat/archivos/WorkingPapers/wp292.pdf

Castaño, María-Soledad; Méndez, María-Teresa; Galindo, Miguel-Ángel (2015). "The effect of social, cultural, and economic factors on entrepreneurship". Journal of business research, v. 68, n. 7, pp. 1496-1500.

https://doi.org/10.1016/j.jbusres.2015.01.040

Castillo-Esparcia, Antonio (2009). “Relaciones públicas y 'think tanks' en América Latina. Estudio sobre su implantación y acción". Razón y palabra, n. 70, pp. 1-22.

http://ww35.razonypalabra.org.mx/N/N70/Castillo_revisado2.pdf

Castillo-Esparcia, Antonio; Guerra-Heredia, Sergio; Almansa-Martínez, Ana (2017). "Political communication and think tanks in Spain. Strategies with the media". El profesional de la información, v. 26, n. 4, pp. 706-713.

https://doi.org/10.3145/epi.2017.jul.14

Chuliá, Elisa (2018). “Una aproximación a los think tanks como organizaciones proveedoras de información y análisis a la sociedad". Revista española de sociología, v. 27, n. 2, pp. 333-340.

https://doi.org/10.22325/fes/res.2018.27

Danielian, Lucig-Hameste (1989). Network news coverage of interest groups: Implications for mass media and democracy. Doctoral dissertation. Austin: University of Texas.

Davis, Aeron (2012). "Mediation, financialization and the global financial crises: An inverted political economy perspective". In: Banks, John; Winseck Dwayne; Yin, Dal-Yong (eds.). The political economies of media: The transformation of the global media industries. London: Bloomsbury Academic, pp. 241-254. ISBN: 9781849668934

Denham, Andrew (2005). British think-tanks and the climate of opinion. London: Routledge. ISBN: 1857284976

España (2012a). "Ley 3/2012, de 6 de julio, de medidas urgentes para la reforma del mercado laboral". BOE, n. 162,7 julio. https://www.boe.es/eli/es/l/2012/07/06/3/dof/spa/pdf

España (2012b). “Memorando de entendimiento sobre condiciones de política sectorial financiera, hecho en Bruselas y Madrid el 23 de julio de 2012, y Acuerdo marco de asistencia financiera, hecho en Madrid y Luxemburgo el 24 de julio de 2012". BOE, n. 296, 10 diciembre.

https://www.boe.es/boe/dias/2012/12/10/pdfs/BOE-A-2012-14946.pdf

Graber, Doris A. (1993). Mass media and American politics. Washington: DC: Congressional Quarterly Press. ISBN: 978 0871876997

James, Simon (1993). "The idea brokers: the impact of think tanks on British government". Public administration, v. 71, n. 4, pp. 491-506.

https://doi.org/10.1111/j.1467-9299.1993.tb00988.x

Lalueza, Ferran; Girona, Ramon (2016). "The impact of think tanks on mass media discourse regarding the economic crisis in Spain". Public relations review, v. 42, n. 2, pp. 271-278.

https://doi.org/10.1016/j.pubrev.2015.09.006

Leeson, Peter T.; Ryan, Matt, E.; Williamson, Claudia R. (2012). "Think tanks". Journal of comparative economics, v. 40, n. 1, pp. 62-77.

https://doi.org/10.1016/j.jce.2011.07.004

Lowery, David; Marchetti, Kathleen (2012). "You don't know Jack: Principals, agents and lobbying". Interest groups \& advocacy, v. 1, n. 2, pp. 139-170.

https://doi.org/10.1057/iga.2012.15

McDonald, Lauren (2014). "Think tanks and the media: How the conservative movement gained entry into the education policy arena". Educational policy, v. 28, n. 6, pp. 845-880.

https://doi.org/10.1177/0895904813492372

McGann, James G. (2007). Think tanks and policy advice in the United States. Academics, advisors and advocates. New York: Routledge. ISBN: 9780415772280

McGann, James G. (2019). 2018 Global go to think tank index report. Philadelphia: University of Pennsylvania. https://repository.upenn.edu/cgi/viewcontent.cgi?article $=1017 \&$ context=think_tanks

McGann, James G.; Viden, Anna; Rafferty, Jillian (2014). How think tanks shape social development policies. Philadelphia: Pennsylvania University Press. ISBN: 9780812246018 
McGann, James G.; Weaver, R. Kent (eds.). (2000). Think tanks \& civil societies: Catalysts for ideas and action. New Brunswick, NJ: Transaction Publishers. ISBN: 9780765809520

Medvetz, Thomas M. (2008). Think tanks as an emergent field. New York: The Social Science Research Council. https://www.ssrc.org/publications/view/think-tanks-as-an-emergent-field

Medvetz, Thomas M. (2012). “Murky power: 'Think tanks' as boundary organizations”. In: Courpasson, David; Golsorkhi, Damon; Sallaz, Jeffrey J. Rethinking power in organizations, institutions, and markets. Emerald Group Publishing Limited, pp. 113-133. ISBN: 9781780526645

https://doi.org/10.1108/S0733-558X(2012)0000034007

Misztal, Barbara A. (2012). "Public intellectuals and think tanks: A free market in ideas?". International journal of politics, culture, and society, v. 25, n. 4, pp. 127-141.

https://doi.org/10.1007/s10767-012-9126-3

Montobbio, Manuel (2013). La geopolítica del pensamiento: Think tanks y política exterior. Madrid: Real Instituto Elcano. http://spain-india.org/files/documentos/87_Montobbio_geopolitica_del_pensamiento_dic2013.pdf

Parrilla, Ricard; Almiron, Núria; Xifra, Jordi (2016). “Crisis and interest: The political economy of think tanks during the great recession". American behavioral scientist, v. 60, n. 3, pp. 340-359.

https://doi.org/10.1177/0002764215613404

Ponsa-Herrera, Francesc (2014). Evolució històrica i models de think tanks a Catalunya. El cas de les fundacions dels partits polítics. Tesis doctoral. Barcelona: Universitat Pompeu Fabra.

https://repositori.upf.edu/handle/10230/22205

Ponsa-Herrera, Francesc; González-Capitel, Jaime (2015). Radiografía de los think tanks en España. Madrid: Funciva. ISBN: 9788494399503

http://oett.es/wp-content/uploads/2017/10/radiograf\%C3\%ADa_de_los_think_tanks_en_espa\%C3\%B1a._2pdf.pdf

Posner, Richard A. (2001). Public intellectuals. A study of decline. Cambridge: Harvard University Press. ISBN: 9780 674012462

Requejo-Coll, Ferran; Jordana-Casajuana, Jacint; Ramió-Matas, Carles; Gil, Eduard (2000). "Els think tanks a Catalunya”. Idees: Revista de temes contemporanis, n. 7, pp. 42-61.

Rich, Andrew (2004). Think tanks, public policy and the politics of expertise. Cambridge, University Press. ISBN: 052183019X

Rich, Andrew; Weaver, R. Kent (2000). "Think tanks in the U.S. media". The international journal of press/politics, v. 5, n. 4, pp. 81-103.

https://doi.org/10.1177/1081180X00005004006

Saura-Casanova, Geo (2015). "Think tanks and education. Faes neoliberalism in the Lomce". Education policy analysis archive, v. 23, n. 107, pp. 1-19.

https://doi.org/10.14507/epaa.v23.2106

Selee, Andrew D. (2013). What should think tanks do?: A strategic guide to policy impact. Stanford: Stanford University Press. ISBN: 9780804787987

Shaw, Sara E.; Russell, Jill; Parsons, Wayne; Greenhalgh, Trisha (2015). "The view from nowhere? How think tanks work to shape health policy". Critical policy studies, v. 9, n. 1, pp. 58-77.

https://doi.org/10.1080/19460171.2014.964278

Sherrington, Philippa (2000). “British think tanks: Advancing the intellectual debate?”. The British journal of politics \& international relations, v. 2, n. 2, pp. 256-263.

https://doi.org/10.1111/1467-856X.00036

Soroka, Stuart N.; Farnsworth, Stephen J.; Lawlor, Andrea; Young, Lori (2013). "Mass media and policymaking". In: Araral, Eduardo; Fritzen, Scott; Howlett, Michael; Ramesh, M.; Wu, Xun. Routledge handbook public policy. Abingdon: Routledge, pp. 1-15. ISBN: 9780415782456

Stone, Diane (1996). Capturing the political imagination: Think tanks and the policy process. London: Frank Cass. ISBN: 0714647160

Stone, Diane (2004). "Introduction: think tanks, policy advice and governance". In: Stone Diane; Denham, Andrew. Think tank traditions. Policy research and the politics of ideas. Manchester: Manchester University Press, pp. 35-50. ISBN: 0719064791

Stone, Diane (2005). "Think tanks and policy advice in countries in transition". Paper prepared for the Asian Development Bank Institute Symposium: How to strengthen policy-oriented research and training in Vietnam. Hanoi: ADB Institute Discussion Papers. 
https://www.adb.org/sites/default/files/publication/156673/adbi-dp36.pdf

Tejerina-Montaña, Benjamín; Perugorría, Ignacia (2017). “La crisis social de la política y la movilización política de la crisis: el 15-M”. Mélanges de la Casa de Velázquez. Nouvelle serie, v. 47, n. 2, pp. 81-99.

https://dialnet.unirioja.es/servlet/articulo?codigo $=6272551$

Tello-Benítez, Marta (2013). Guía de think tanks en España. Valencia: Centro Francisco Tomás y Valiente; UNED Alzira-Valencia. ISBN: 9788492885213

http://www.funciva.org/descargas/libros/guia-think-tanks-espana-segunda-edicion-corregida-aumentada-2

Urrutia, Olivier (2013). "El papel de los think tanks en la definición y aplicación de las políticas y estrategias de defensa”. Revista del Instituto Español de Estudios Estratégicos, n. 2, pp. 1-34.

http://revista.ieee.es/article/view/340

Wiarda, Howard J. (2008). "The new powerhouses: Think tanks and foreign policy". American foreign policy interests, v. 30, n. 2, pp. 96-117.

https://doi.org/10.1080/10803920802022704

Xifra, Jordi (2005). "Los think tank y advocacy tank como actores de la comunicación política". Anàlisi: quaderns de comunicació i cultura, n. 32, pp. 73-91.

https://www.raco.cat/index.php/Analisi/article/view/15173/179893

\section{Colección de libros de bolsillo El profesional de la información (Editorial UOC) Últimos títulos publicados}
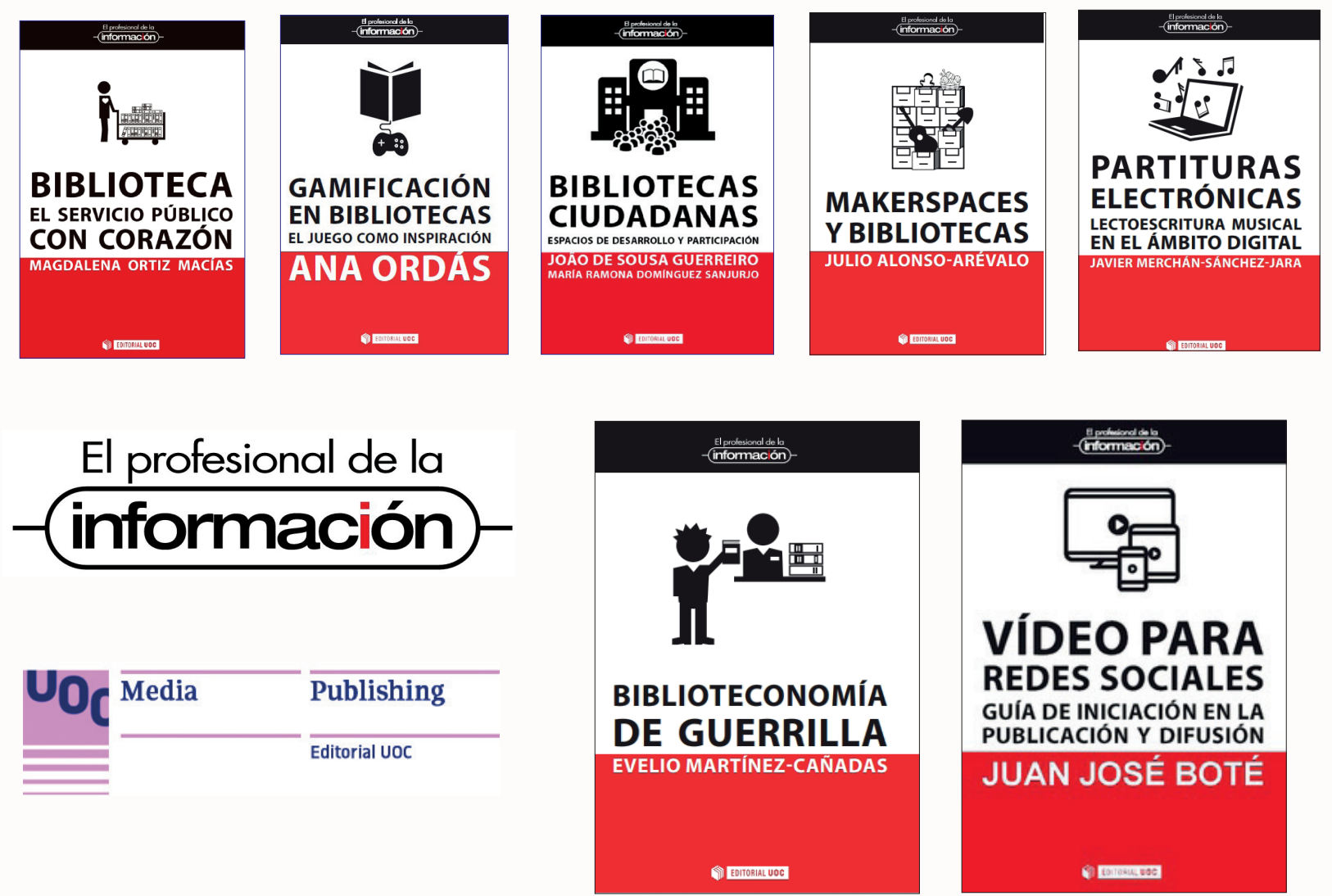

Más información:

http://www.elprofesionaldelainformacion.com/libros.html 